\author{
Patrick PÖHLMANN ${ }^{1 *}$ \\ Christoph PEUKERT ${ }^{1}$ \\ Marcel MERX ${ }^{1}$ \\ Jens MÜLLER ${ }^{1}$ \\ Steffen IHLENFELDT ${ }^{1,2}$
}

\title{
COMPLIANT JOINTS FOR THE IMPROVEMENT OF THE DYNAMIC BEHA VIOUR OF A GANTRY STAGE WITH DIRECT DRIVES
}

\begin{abstract}
Gantry stages, which consist of two parallel acting servo drives, are commonly used in machine tools. One drawback of this concept is the crosstalk between both drives due to the structural coupling that can cause stability issues and therefore limits the bandwidth of the position control. This paper deals with the development of compliant joints to solve the coupling between the drives. When compared to solutions containing bearings, the advantages of such flexible elements are low friction and the absence of backlash. To adjust the properties of the joints, packages of spring-steel-sheets are used as compliant links. One design aspect of the flexible joints is a low stiffness relating to the rotation around one specific axis, but a high stiffness relating to the other degrees of freedom. With this method, the dynamic behaviour of the gantry stage is modified and the bandwidth of the controllers can be increased. Additionally, by releasing the mechanical coupling of the drives, the reaction forces the actuators have to provide can be reduced. Both systems with flexible and with rigid connecting elements, are analysed by measured frequency response functions.
\end{abstract}

\section{INTRODUCTION}

Compliant joints are a well established alternative to common roller bearings or linear guides when only small rotations or linear movements are required. The main advantages are not only the low friction and the absence of backlash, but also their compact design. With these benefits, many different applications have been developed. Compliant mechanisms are frequently used for microelectromechanical systems (MEMS) in actuators and sensors, as shown in [1]. The application of a miniature gripper for cell manipulation and bulk stiffness estimation described in [2] or a crash sensor that is based on a compliant bistable mechanism [3] are such examples.

\footnotetext{
${ }^{1}$ Technische Universität Dresden, Faculty of Mechanical Science and Engineering, Institute of Mechatronic Engineering, Chair of Machine Tools Development and Adaptive Controls, Dresden, Germany

${ }^{2}$ Fraunhofer Institute for Machine Tools and Forming Technology IWU, Dresden, Germany

*E-mail: patrick.poehlmann@tu-dresden.de https://doi.org/10.36897/jme/127103
} 
Another field of application is the design of high-precision positioning stages. There are examples with one and two degrees of freedom (DoF) (see e.g. [4], [5]) or even six DoF movability [6]. Mechanisms with three DoF for planar movement ( $X Y \theta$, see e.g. [7]) and three translational DoF $(X Y Z)$ are investigated in [8] or [9]. In these studies, both serial [4], [8] and parallel [5-7], [9] mechanism structures are designed and piezoelectric actuators are often used. Other applications are for example clamping mechanisms for tool holders [10] or part assembling and insertion units [11].

This work focuses on a gantry stage with two parallel acting direct drives. This is a common concept in machine tool design since high accelerations can be achieved as well as a split of the loads and, therefore, reduced local actuator forces. Nevertheless, if the mechanical coupling between the drives cannot be neglected, challenges for the control design will arise. Challenges might be stability issues, caused by the interaction between the drives. In the event of a very stiff mechanical coupling, an equal split of the actuator forces is not guaranteed, and the full power capability of each drive will not be reached. The replacement of the classical master-master-controller by concepts that consider the interaction between the drives is a common approach to tackle these issues [12]. State space approaches, sliding mode controllers [13] or modal decoupling [14] are some examples of this group. An important requirement of these strategies is the exchange of information between the different drives with short cycle times and low delay to achieve a high bandwidth of the closed loop. However, independent control loops (master-master) are the most common solution in industrial applications. The direct integration of the controllerfunctionality in the inverter, results in quick signal processing.

In this paper, the conventional master-master-controller, with cascaded velocity - and position controllers, is used to control the gantry stage with two linear motors. The integration of flexible joint-elements resolves the mechanical coupling of the drives. First, the test bed is described in Section 2, followed by the design of the compliant elements in Section 3. A disadvantage of compliant mechanisms is a high stress concentration and a reduced lifetime [15]. To deal with this challenge, spring steel sheets are used as elastic links, which enable the modification of the properties of the joints by replacing the sheets. The effect of this design approach on the dynamic behaviour and the performance of the control loop is investigated by measured frequency response functions (FRF) in Section 4. In previous work, numeric analyses have revealed an improvement of the controller bandwidth by using compliant joints [16]. The aim of this work is to verify these results experimentally.

\section{DESCRIPTION OF THE TEST BED}

Figure 1 pictures the whole test rig with two independent gantry stages, which has already been introduced in previous contributions (e.g. [17]). The moving part of each stage consists of three main parts: two separate drive units at both sides left and right, and a bridge plate in between. Each drive unit is driven by a linear motor (Tecnotion UXX6N) with fixed permanent magnets (magnet yokes) and is guided by two carriages, mounted on the guiding rail. The guide rails have a cross-axial distance of $770 \mathrm{~mm}$. Two absolute linear 
measurement systems (Heidenhain LIC4117) determine the positions of each drive unit with an accuracy of $\pm 5 \mu \mathrm{m}$ and a resolution of $1 \mathrm{~nm}$. In this configuration, rigid coupling elements connect the bridge to the drive units. These are replaced by the compliant elements, which will be described in the next section. The bridge, the connecting elements and the drive units are mainly made from aluminium. The moving stages have a total weight of $29.4 \mathrm{~kg}$ each. Only one of the gantry stages is used in the following investigations.

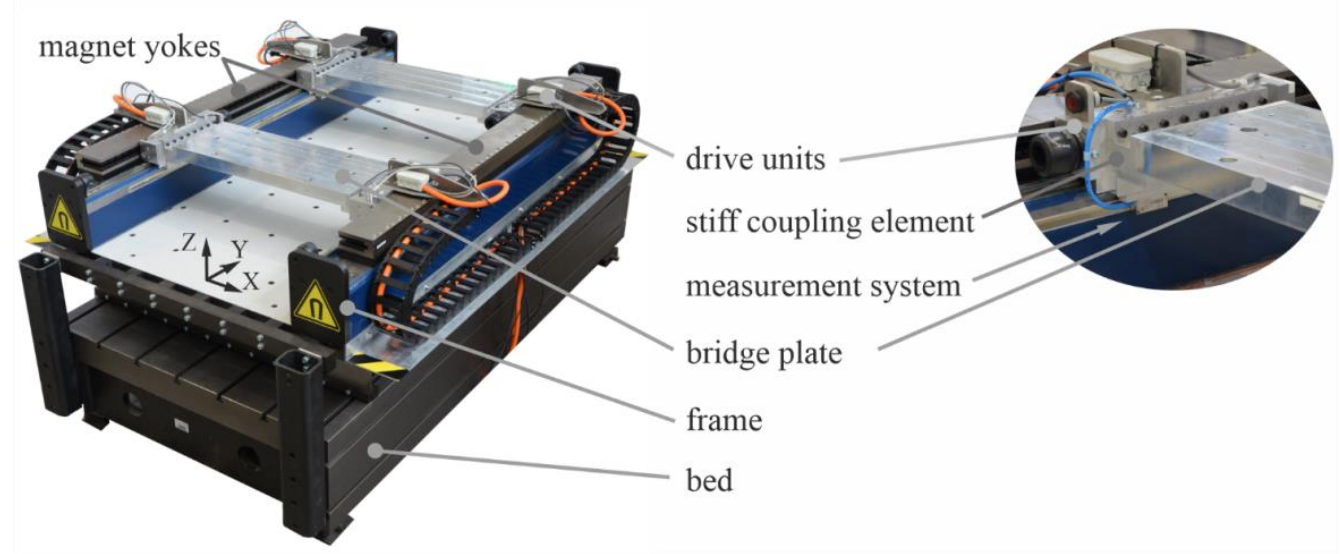

Fig. 1. Test bed with two linear motor driven gantry stages

\section{DESIGN OF THE FLEXIBLE JOINTS}

\subsection{DESIGN PRINCIPLES}

With the large number of applications for compliant joints and mechanisms, many different design techniques have been established. Howell [18] gives an overview of the most used concepts. One example is the idea of using an existing rigid-body mechanism and replacing the joints by compliant links. This provides the opportunity to use methods for the synthesis and analysis of rigid mechanisms, which are well known in the literature. The compliant links are often created by manufacturing notches in the rigid mechanical structure of the compliant joint. These notches can have different shapes like circles (see e.g. [5], [7]), ellipses and others [19]. Topology optimisation, by contrast, is a method to find a solution by numerical calculation. An example is given in [15], where the maximal geometric area and the boundary conditions of the mechanism are defined in the first step. This area is filled with an array of nodes that are connected to each other with bundles of beams. An optimisation algorithm reduces the number of connectors using a suitable objective function and the mechanism is generated automatically. However, since a lot of investigations and examples concerning the design of flexible joints had already been made, the selection and modification of an existing solution is an efficient way to design a flexible mechanism for a specific task. In each case, the consideration of different design concepts is important, because the decision about which method to use is an important design-aspect and a combination is possible as well [18]. 
Hopkins and Culpepper introduce the approach of Freedom and Constraint Topology (FACT), which is a general approach to synthesise compliant constraints for a permissible motion as well as to analyse the DoFs of a given system [20]. This method is based on sets of constraint lines, which build the constraint space and sets of freedom lines, and which build the freedom space. A main principle of this method is the direct relationship between these spaces. To design a compliant concept for a given problem, the desired motion and the freedom space of the system are defined in the first step. Then the space of all possible constraints is mapped on this freedom space. A set of constraint lines can be chosen, whereby each non-redundant constraint removes one DoF from the system. Additional redundant lines can be chosen as well to optimise parameters such as stiffness, load capacity or symmetry.

\subsection{DESIGN OF THE COMPLIANT JOINTS}

The main objective of the compliant joints is to generate an additional degree of freedom for small rotations $\theta$ of the stage around the vertical $Z$-axis by positioning the drive units with a relative displacement $y_{\text {rel }}$. The idea is to replace the rigid connection elements (Fig. 2a) by rotational joints, which is displayed in Fig. 2 b as a rigid-body model. The available space for the joints is limited to a cuboid with a length of $260 \mathrm{~mm} Y$, a width of $55 \mathrm{~mm} X$ and a height of $58 \mathrm{~mm} \mathrm{Z}$. Figure 2 shows the rotational axis (freedom space) and a selection of planes that represent constraints (constraint space) between the drive units and the bridge plate at one side of the stage [20]. A set of these planes is chosen and replaced by flexible links.
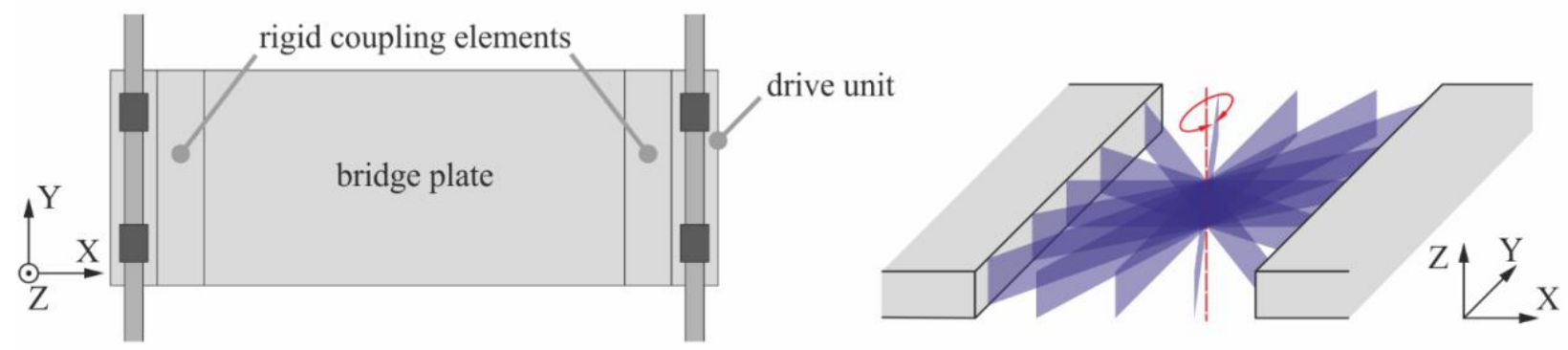

a) gantry stage with rigid coupling elements

c) constraints and DoF of a rotaional joint
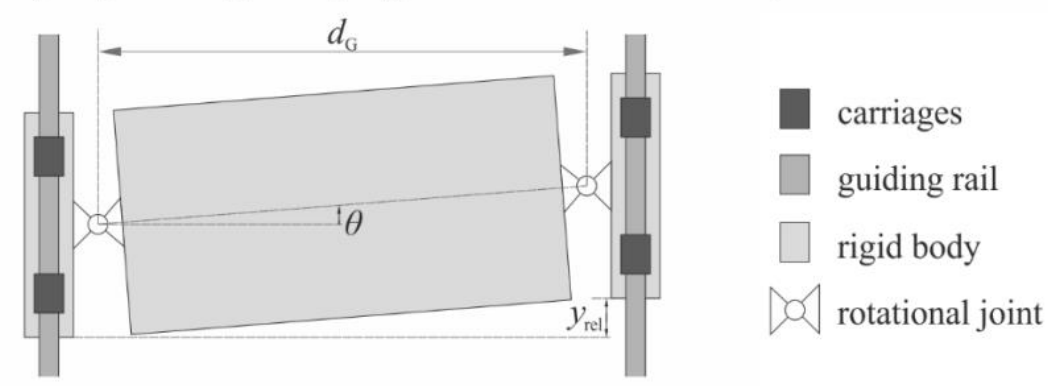

b) gantry stage with rotational joints

Fig. 2. Concept of the joint design 
Figure 3 illustrates the structure of the designed joint. It consists of two connection plates for the attachment to the drive unit and the bridge as well as a couple of clamping blocks to hold the flexible links. One main advantage of this concept is that the parts can be fixed in different arrangements and, therefore, parameters like the length (see Fig. 3c) or thickness of the spring steel sheets can be varied and evaluated. The steel sheets are placed parallel to the $Y$-Z-plane A, C and the $X$-Z-plane B which results in high stiffness in the $X$ - and $Y$-direction. The parts are clamped together with numerous screws to achieve a high contact ratio and stiffness (see Fig. 3a).

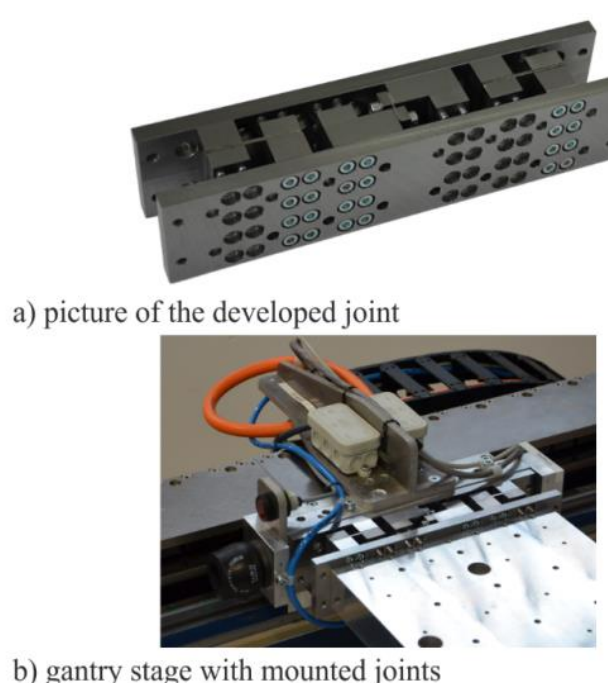

b) gantry stage with mounted joints

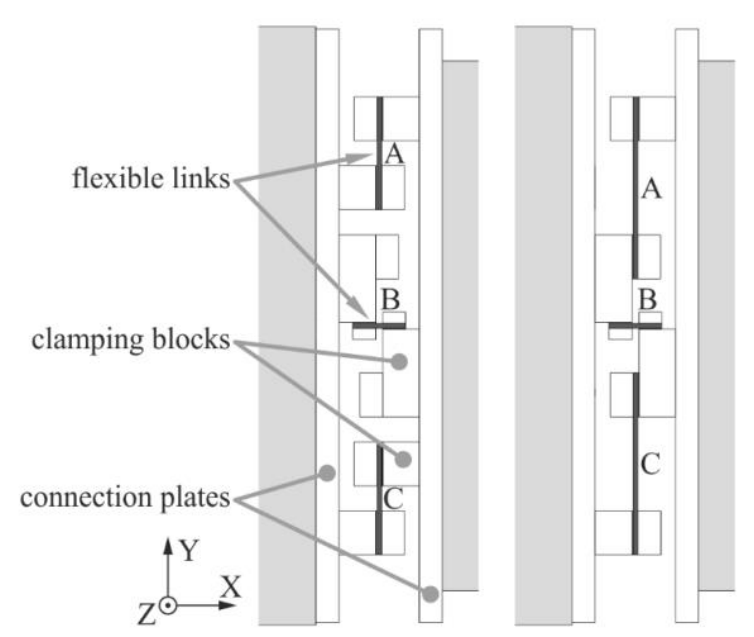

c) joints with short (left) and long (right) links A and C

Fig. 3. Joints for the experimental investigation

Instead of single sheets, packages of multiple thin sheets can be used as well. In the case of the links parallel to the $Y$-Z-plane A, C, packaging multiple thin sheets leads to a reduced overall rotational joint stiffness about the $Z$-axis compared to the use of single sheets with the same thickness as the package. This is due to reduced translational stiffness of a package compared with a single steel sheet, since the links $\mathrm{A}$ and $\mathrm{C}$ mainly experience a displacement, rather than a rotation. The stiffness in $Y$-direction does not change significantly, because the cross section of the link does not change. Table 1 lists the dimensions and the number of sheets of the flexible links for the investigated configurations, where the same length and thickness of the links are used. The first configuration consists of one single sheet in the links $\mathrm{A}$ and $\mathrm{C}$ whereas the second one consists of ten thin sheets. In both cases, the configuration with short links $\mathrm{A}$ and $\mathrm{C}$ (see Fig. 3c, left) with a free bending length of $11.0 \mathrm{~mm}$ is used.

Table 1. Dimensions of the flexible links

\begin{tabular}{|c|c|c|c|c|c|c|}
\hline $\begin{array}{c}\text { Link } \\
\text { configuration }\end{array}$ & Flexible link & $\begin{array}{c}\text { Number of } \\
\text { sheets }\end{array}$ & $\begin{array}{c}\text { Thickness per } \\
\text { sheet }\end{array}$ & $\begin{array}{c}\text { Total } \\
\text { thickness }\end{array}$ & Free length & Height $(Z)$ \\
\hline \multirow{2}{*}{$1-$ one sheet } & B & 1 & $1.0 \mathrm{~mm}$ & $1.0 \mathrm{~mm}$ & $3.0 \mathrm{~mm}$ & $58.0 \mathrm{~mm}$ \\
\cline { 2 - 7 } & A, C & 1 & $2.0 \mathrm{~mm}$ & $2.0 \mathrm{~mm}$ & $11.0 \mathrm{~mm}$ & $58.0 \mathrm{~mm}$ \\
\hline \multirow{2}{*}{$2-$ package } & B & 1 & $1.0 \mathrm{~mm}$ & $1.0 \mathrm{~mm}$ & $3.0 \mathrm{~mm}$ & $58.0 \mathrm{~mm}$ \\
\cline { 2 - 7 } & A, C & 10 & $0.2 \mathrm{~mm}$ & $2.0 \mathrm{~mm}$ & $11.0 \mathrm{~mm}$ & $58.0 \mathrm{~mm}$ \\
\hline
\end{tabular}


The mechanical structure of the joints is modelled with the FE-software ANSYS. Fig. 4 shows the meshed structure of one joint element as well as one single sheet, where hexahedrons are used for all elements. When modelling the steel-sheet-packages, it has to be taken into account whether a section is clamped or is able to bend freely. In the free part of the sheets, no contact relation is appended between nearby surfaces.

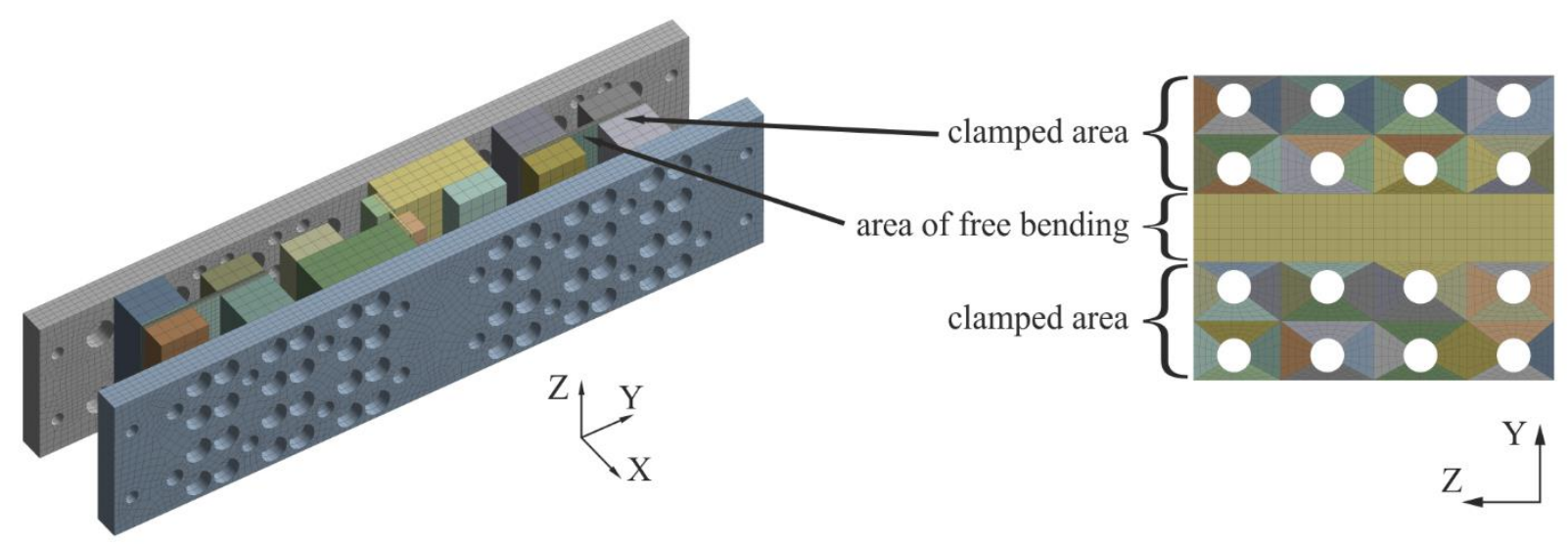

Fig. 4. FE-model of the joint element (left) and a single sheet (right)

The FE model of the full gantry stage includes two of the coupling elements as well as models of the drive units and the bridge plate. Linear springs are integrated to consider the coupling between the carriages and the guide rails. The stiffness of the springs is adjusted to get a high similarity to the dynamic behavior of the real stage, which is described in the next section. A modal analysis is carried out for each of the configurations 0 with stiff coupling elements as well as 1 and 2 with the joints. This shows that the system dynamic range is mainly dominated by the rigid body movement of the full stage as well as the first eigenmode that represents the rotation of the bridge around the $Z$-axis. Fig. 5 (left) shows the shape of the second mode for configuration 2. The next mode shape that has notable influence on the dynamics of the stage is displayed as well. Table 2 lists the eigen-frequencies of these modes. Since the shapes are different for all configurations (especially configuration 0 ), the modes with the best conformity are considered. In the case of the rigid configuration 0 , the second mode is mainly influenced by the stiffness of the carriages. This frequency is reduced significantly with the use of compliant elements since rotation of the bridge relative to the drive units is enabled.
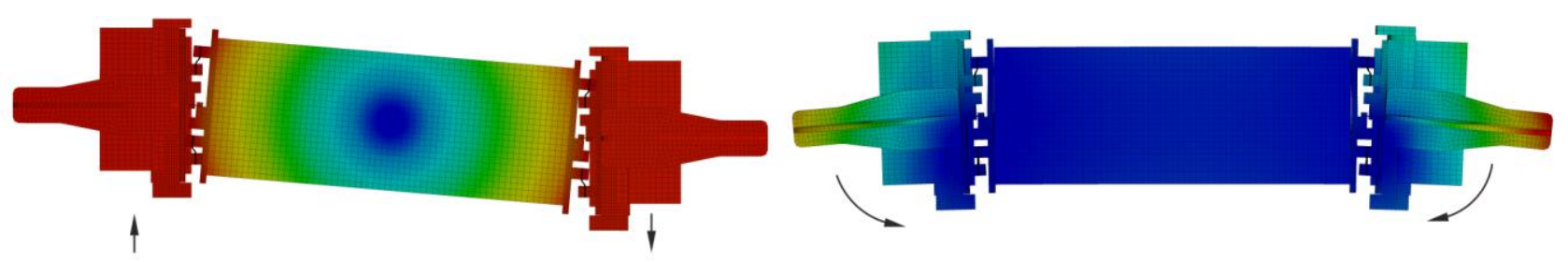

Fig. 5. Second and third mode shape of the gantry stage for configuration 2 calculated with the FE-model 
The analyses show that other frequencies (e.g. mode 3) are reduced as well. This has to be considered in any further investigations. However, the frequency response measurements in the following section show that the resonance peaks of configuration 1 and 2 are small in the frequency range of the simulated third modes $(500 \mathrm{~Hz}-700 \mathrm{~Hz})$.

Table 2. Eigenfrequencies of the first eigenmodes of the FE-model of the gantry stage

\begin{tabular}{|l|c|c|c|}
\hline Configuration & $1^{\text {st }}$ Mode & $2^{\text {nd }}$ Mode & $3^{\text {rd }}$ Mode \\
\hline $0-$ rigid & $0 \mathrm{~Hz}$ & $168 \mathrm{~Hz}$ & $1215 \mathrm{~Hz}$ \\
\hline $1-$ one sheet & $0 \mathrm{~Hz}$ & $65 \mathrm{~Hz}$ & $615 \mathrm{~Hz}$ \\
\hline $2-$ package & $0 \mathrm{~Hz}$ & $15 \mathrm{~Hz}$ & $567 \mathrm{~Hz}$ \\
\hline
\end{tabular}

\section{EXPERIMENTAL INVESTIGATIONS}

The main aspect of this study is to examine the influence of compliant joint elements in comparison to a rigid attachment of the stage bridge plate. The following sections deal with the performance of the cascaded velocity and position control. The results are based on frequency response function (FRF) measurement of the closed loop system.

\subsection{SYSTEM IDENTIFICATION}

With the two forces $f_{1}$ and $f_{2}$ of the direct drives and the two velocity values $v_{1}$ and $v_{2}$, the gantry stage can be described by a matrix $\boldsymbol{G}(\mathrm{j} \omega)$ consisting of four FRFs:

$$
\underbrace{\left(\begin{array}{l}
v_{1}(\mathrm{j} \omega) \\
v_{2}(\mathrm{j} \omega)
\end{array}\right)}_{\boldsymbol{v}(\mathrm{j} \omega)}=\underbrace{\left(\begin{array}{ll}
G_{11}(\mathrm{j} \omega) & G_{12}(\mathrm{j} \omega) \\
G_{21}(\mathrm{j} \omega) & G_{22}(\mathrm{j} \omega)
\end{array}\right)}_{\boldsymbol{G}(\mathrm{j} \omega)} \cdot \underbrace{\left(\begin{array}{l}
f_{1}(\mathrm{j} \omega) \\
f_{2}(\mathrm{j} \omega)
\end{array}\right)}_{\boldsymbol{f}(\mathrm{j} \omega)},
$$

where $v_{1}$ and $v_{2}$ are the time derivatives of the linear encoder signals $y_{1}$ and $y_{2}$. To determine the entries of $\boldsymbol{G}(\mathrm{j} \omega)$, the system is excited with pseudo random binary sequence PRBS signals. The resulting displacement-response is measured at both sides and the FRFs are calculated subsequently. To avoid issues arising from the static friction of the guiding system, a low bandwidth motion controller keeps the stage in continuous motion. It need to be noted that the forces that act on the system are not measured directly. The given values in this and the following sections are calculated from the electric current by consideration of the force constant of the drives of 124 N/A. Figure 6 depicts $G_{11}(\mathrm{j} \omega)$ (left) and $G_{21}(\mathrm{j} \omega)$ (right) for configuration 0 with rigid elements as well as configuration 1 and 2 with compliant joints. Since the system is symmetric, $G_{22}(\mathrm{j} \omega)$ is almost equal to $G_{11}(\mathrm{j} \omega)$ and $G_{12}(\mathrm{j} \omega)$ is almost equal to $G_{21}(\mathrm{j} \omega)$ and their depiction is omitted as a consequence.

The amplitudes of $G_{11}(\mathrm{j} \omega)$ and $G_{21}(\mathrm{j} \omega)$ of configuration 0 are dominated by a straight line with a negative slope of $10(\mathrm{~m} / \mathrm{s}) / \mathrm{N}$ per decade which corresponds to the rigid body 
motion of the stage for low frequencies. Replacing the rigid connecting elements with flexible joints leads to a reduction of the first eigenfrequency (see also Fig. 5 and Table 2), depending on the reduced torsional stiffness of the element.
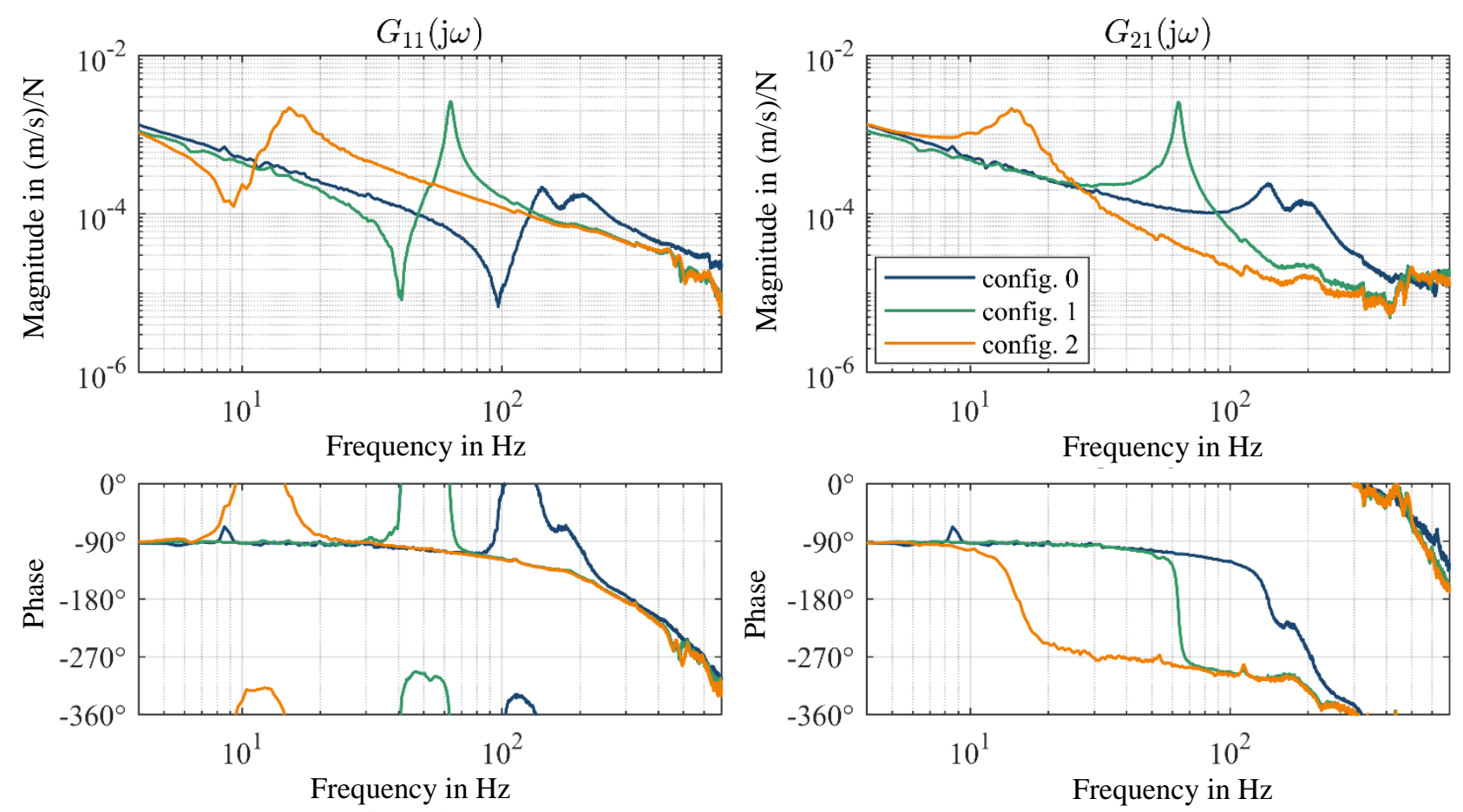

Fig. 6. FRFs of the open loop system

\subsection{REACTION FORCES}

One drawback of a stiffly coupled gantry stage is the high reaction forces to the drives and the guiding elements arising from small geometric inaccuracies. These lead to a reduced lifetime of the components as well as high actuator forces and power consumption. The inverse matrix of $1 /(\mathrm{j} \omega) \cdot \boldsymbol{G}(\mathrm{j} \omega)$ (see Section 4.1) gives the relation between displacements and the resulting forces in the frequency domain:

$$
\left(\begin{array}{l}
f_{1}(\mathrm{j} \omega) \\
f_{2}(\mathrm{j} \omega)
\end{array}\right)=(1 /(\mathrm{j} \omega) \cdot \boldsymbol{G}(\mathrm{j} \omega))^{-\mathbf{1}} \cdot\left(\begin{array}{l}
y_{1}(\mathrm{j} \omega) \\
y_{2}(\mathrm{j} \omega)
\end{array}\right)=\boldsymbol{G}^{*}(\mathrm{j} \omega) \cdot\left(\begin{array}{l}
y_{1}(\mathrm{j} \omega) \\
y_{2}(\mathrm{j} \omega)
\end{array}\right)
$$

The actuator forces arising with a relative displacement $y_{\text {rel }}(\mathrm{j} \omega)=y_{1}(\mathrm{j} \omega)-y_{2}(\mathrm{j} \omega)$ between the drives are calculated from the differences between the elements of $\boldsymbol{G}^{*}(\mathrm{j} \omega)$ :

$$
\begin{aligned}
& f_{1}(\mathrm{j} \omega) / y_{\text {rel }}(\mathrm{j} \omega)=\left(G_{11}^{*}(\mathrm{j} \omega)-G_{12}^{*}(\mathrm{j} \omega)\right), \\
& f_{2}(\mathrm{j} \omega) / y_{\text {rel }}(\mathrm{j} \omega)=\left(G_{21}^{*}(\mathrm{j} \omega)-G_{22}^{*}(\mathrm{j} \omega)\right) .
\end{aligned}
$$

The magnitude of the function $f_{1}(\mathrm{j} \omega) / y_{\mathrm{rel}}(\mathrm{j} \omega)$ is calculated for the three configurations and displayed in Fig. 7. The curves converge to horizontal lines for low 
frequencies. The level of these lines represents the static stiffness for the relative displacement. This value is reduced significantly when replacing the rigid configuration 0 by configuration 1 and even more for configuration 2 using packaged spring steel sheets.

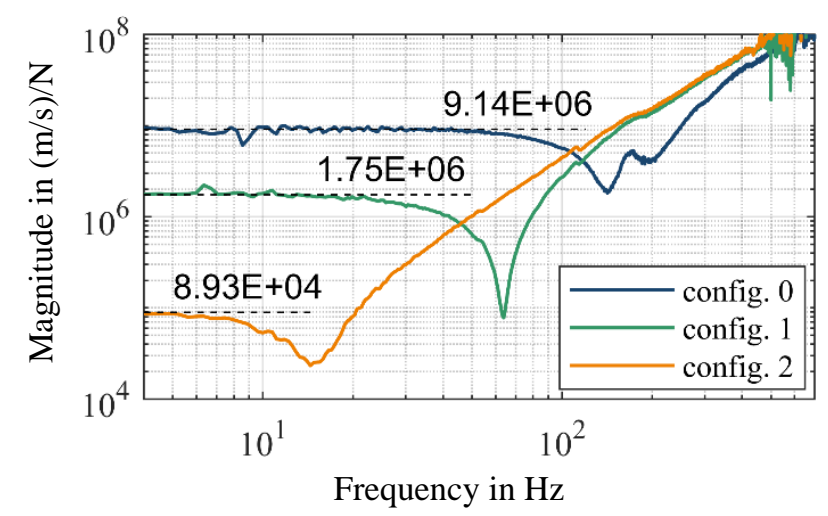

Fig. 7. Actuator reaction forces $f_{1}(\mathrm{j} \omega)$ plotted over $y_{\text {rel }}(\mathrm{j} \omega)$

\subsection{CONTROL}

The control of each drive is performed independently (master-master-control) by cascaded velocity (PI) and position (P) controllers, which are commonly used in industrial feed-drive applications. The controller gains of both drives are set to the same values in each case. The behaviour of the velocity controller is analysed in the first step. The velocity controller $\boldsymbol{R}$ is defined by:

$$
\boldsymbol{R}=\left(\begin{array}{cc}
R_{11} & 0 \\
0 & R_{22}
\end{array}\right), \text { where } R_{11}=R_{22}=K_{\mathrm{P}}\left(1+\frac{1}{T_{\mathrm{N}} \cdot S}\right) .
$$

To obtain a valid comparison of the closed-loop-performance between the different configurations, the parameters of the control-loops are tuned with the same procedure. The gain of the velocity controller $K_{\mathrm{P}}$ is raised up to the critical value (integrator is not active), where the closed loop starts to oscillate. The critical gain-value is divided by two and the time constant $T_{\mathrm{N}}$ is set to $10 \mathrm{~ms}$ in each case. The gains found for configuration 0,1 and 2 are given in Table 3 and are called setups $0,1 b$ and $2 b$ here. The controller setups 1a and $2 \mathrm{a}$ for the configurations 1 and 2 use the same values that are found for configuration 0 . To have a closer look at the stability margins of the velocity controller, the open loop is analysed with the Nyquist criterion. Since the plant is a multivariable system, the eigenvalues $\lambda_{1}(\mathrm{j} \omega)$ and $\lambda_{2}(\mathrm{j} \omega)$ of the open loop matrix $\boldsymbol{G R}$ are considered [21]. These are displayed in Fig. 8 as functions of frequency. Since the controller function is equal for each drive, the gain $K_{\mathrm{P}}$ works as a scalar factor on the open loop and its eigenvalues. The critical gain is reached, when the magnitude of one of the eigenfunctions is equal to 1.0 with a phase value of $-180^{\circ}$, which occur in a frequency range from $239 \mathrm{~Hz}$ to $269 \mathrm{~Hz}$ in $\lambda_{1}$ for each of the controller setups. The gain margins of the velocity controllers are given in 
Table 3 as well. On the one hand, the use of compliant joints (setup 1a and 2a) results in significantly higher gain margins, when the same parameters are used as the rigid system (setup 0). On the other hand, it is possible to use higher gains and keep an acceptable gain margin (see set. $1 \mathrm{~b}$ and $2 \mathrm{~b}$ ). This is mainly caused by the overshoot in the magnitude at the first eigenfrequency of the rigid system (configuration 0 ), which reduces the achievable gain margin (see Fig. 8, $\lambda_{1}$ ). The integration of the flexible joints shifts the first eigenmode to lower frequencies, where a higher phase margin is present.
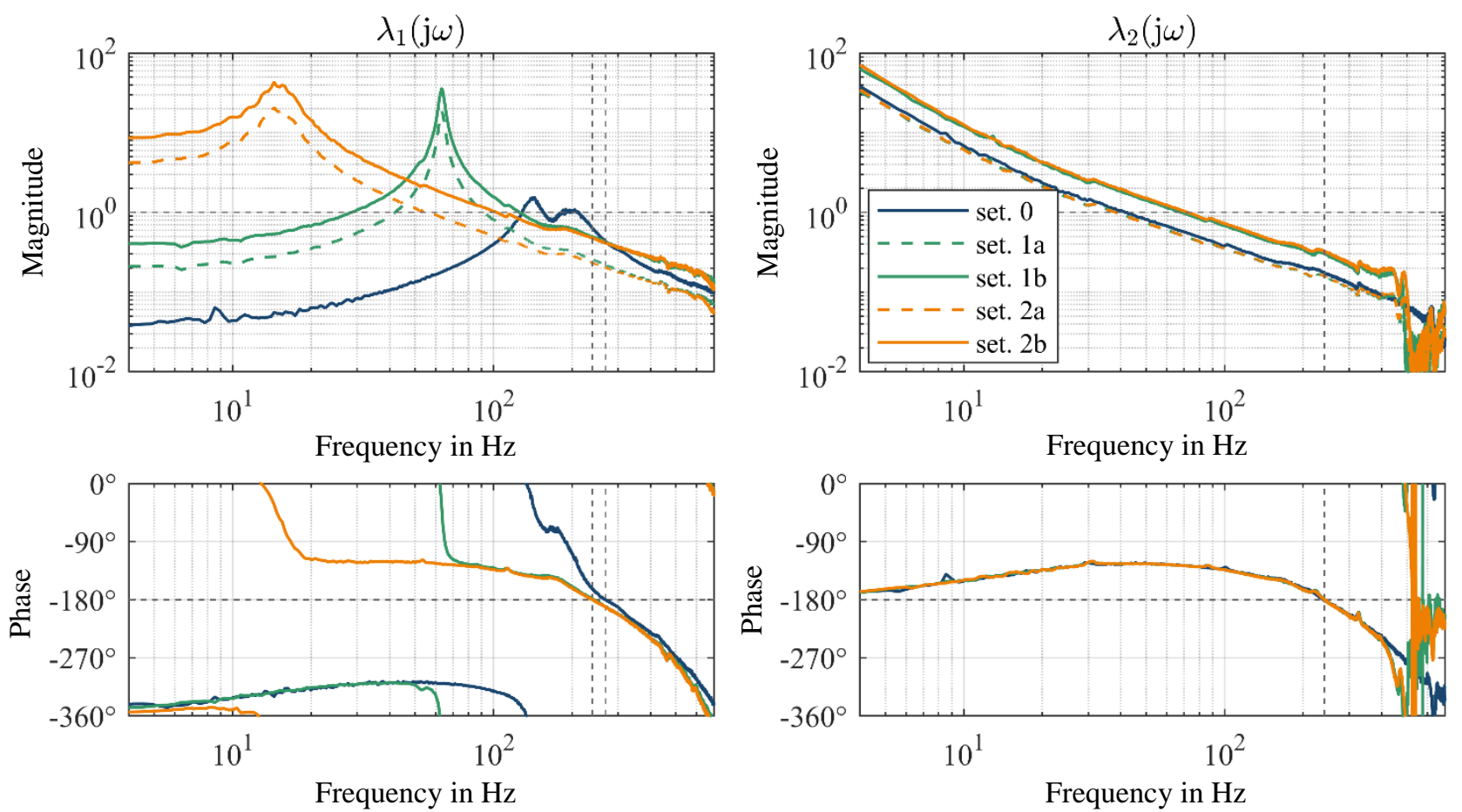

Fig. 8. Eigenfunctions $\lambda_{1}(\mathrm{j} \omega)$ and $\lambda_{2}(\mathrm{j} \omega)$ of the open loop matrix $\boldsymbol{G} \boldsymbol{R}$ of the velocity control
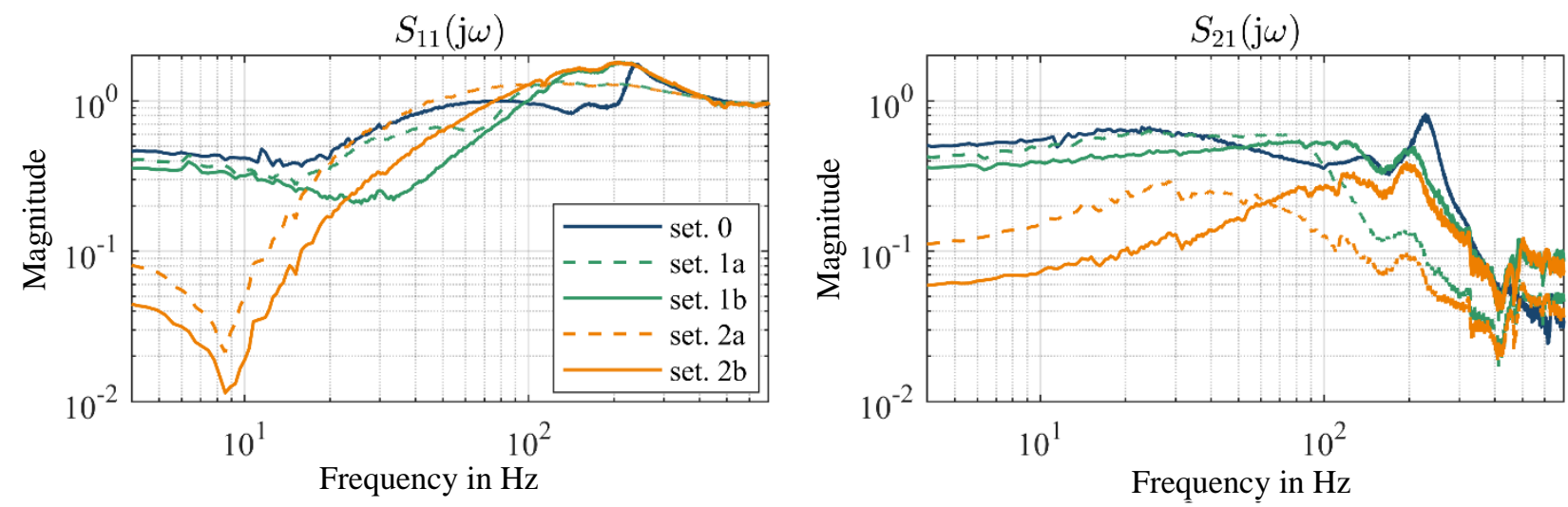

Fig. 9. Sensitivity functions of the closed velocity control loop

Fig. 9 depicts the sensitivity matrix $\boldsymbol{S}(\mathrm{j} \omega)$ that describes the response of the system to a noise signal at the output:

$$
\boldsymbol{S}=(\mathbf{I}+\boldsymbol{G} \boldsymbol{R})^{-1},
$$


where $\mathbf{I}$ is the identity matrix. To achieve a low amplification of the disturbances at the output, the magnitudes of the entries of $\boldsymbol{S}(\mathrm{j} \omega)$ are required to be small. The controller setup 0 for the rigid system shows an overshoot in $S_{11}(\mathrm{j} \omega)$ and $S_{21}(\mathrm{j} \omega)$ at about $250 \mathrm{~Hz}$, which is not present in setup 1a and $2 \mathrm{a}$ where the same gains are used for the flexible systems. When the gains are raised up (setup $1 \mathrm{~b}$ and $2 \mathrm{~b}$ ) the magnitudes get smaller for low frequencies but the maximum values between $100 \mathrm{~Hz}$ and $250 \mathrm{~Hz}$ in $S_{11}(\mathrm{j} \omega)$ rise. The response functions of the system to a disturbance signal at the input

$$
\boldsymbol{G}_{\mathrm{d}}=(\mathbf{I}+\boldsymbol{G} \boldsymbol{R})^{-1} \boldsymbol{G},
$$

are printed in Fig. 10 where the flexible joints reduce the magnitude of $G_{\mathrm{d}, 11}(\mathrm{j} \omega)$ over a wide range of frequencies and particularly for higher gains.
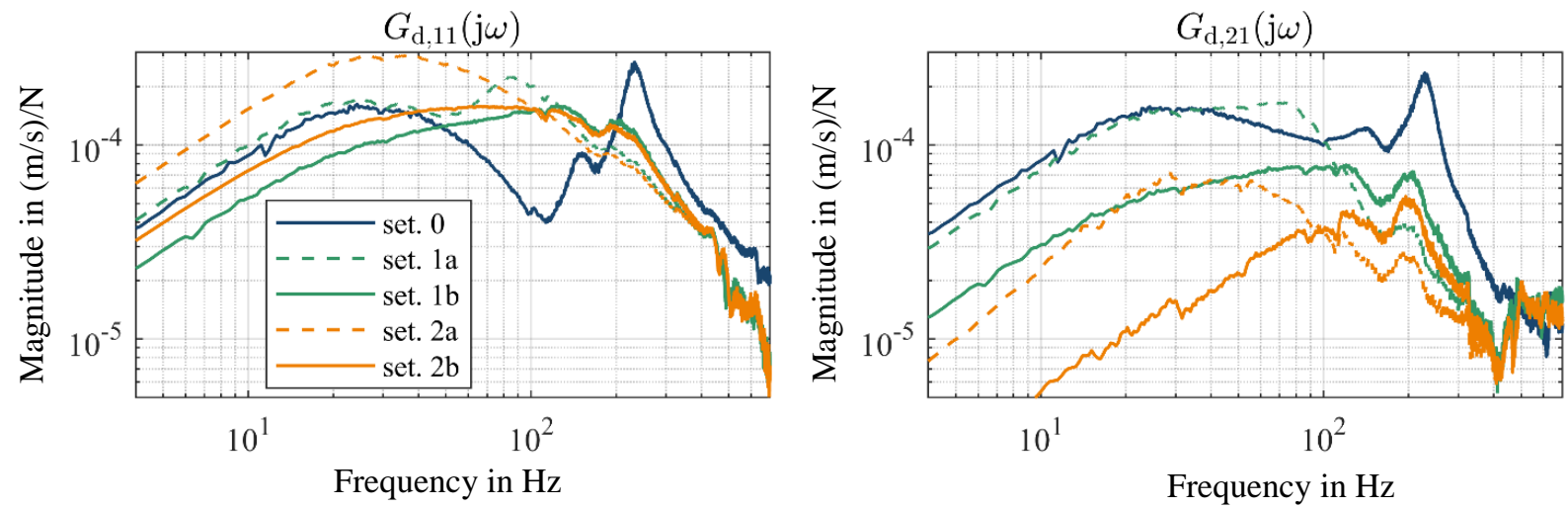

Fig. 10. Disturbance behaviour of the closed velocity control loop

Finally, the closed loop response functions are shown in Fig. 11 for both closed velocity loop $G_{\mathrm{cv}}(\mathrm{j} \omega)$ (left) and closed position loop $G_{\mathrm{cp}}(\mathrm{j} \omega)$ (right). The setpoint values are given to both drives equally to achieve a translational motion. The gain $K_{\mathrm{V}}$ of the position controller is raised up until the transfer function hits the $0 \mathrm{~dB}$ line with no overshoot (setup $0,1 b$ and $2 b$ ). In setup $1 \mathrm{a}$ and $2 \mathrm{a}$, the same values are used as for setup 0 and the results in Fig. 11 are nearly equal. Since the bandwidth of the velocity controller gets wider with higher gains, a wider bandwidth is also achieved in the overlaying position control loop. However, the difference between configuration 1 and 2 is negligible, for low gains (setup 1a and $2 \mathrm{a}$ ) and high gains (setup $1 \mathrm{~b}$ and $2 \mathrm{~b}$ ). This results from the fact that the magnitude of the eigenvalues at the critical frequency does not change significantly by shifting the eigenfrequency form $65 \mathrm{~Hz}$ down to $15 \mathrm{~Hz}$ (see Fig. 8).

Table 3. Parameters of the velocity and position control

\begin{tabular}{|c|c|c|c|c|c|}
\hline \multirow{2}{*}{ Setup } & \multirow{2}{*}{ Configuration } & \multicolumn{3}{|c|}{ Velocity - PI } & Position $-\mathrm{P}$ \\
\cline { 3 - 6 } & & $K_{\mathrm{P}}$ in $\mathrm{A} /(\mathrm{m} / \mathrm{s})$ & $T_{\mathrm{N}}$ in $\mathrm{ms}$ & gain marg. $\lambda_{1}$ & $K_{\mathrm{V}}$ in $1 / \mathrm{s}$ \\
\hline 0 & $0-$ rigid & 28.5 & 10.0 & $7.22 \mathrm{~dB}$ & 108 \\
\hline $1 \mathrm{a}$ & $1-$ one sheet & 28.5 & 10.0 & $11.96 \mathrm{~dB}$ & 108 \\
\hline $1 \mathrm{~b}$ & $1-$ one sheet & 55.0 & 10.0 & $6.25 \mathrm{~dB}$ & 190 \\
\hline $2 \mathrm{a}$ & $2-$ package & 28.5 & 10.0 & $12.72 \mathrm{~dB}$ & 108 \\
\hline $2 \mathrm{~b}$ & $2-$ package & 58.5 & 10.0 & $6.48 \mathrm{~dB}$ & 200 \\
\hline
\end{tabular}


It needs to be considered that this improvement is not always valid if a stiff structure is weakened. If higher eigenmodes are shifted to lower frequencies too, they may lead to stability issues as well. The advantage of the flexible joints is that only the first eigenfrequency is influenced significantly, since compliance is restricted to one DoF and stiffness concerning other DoFs is kept at a high value.
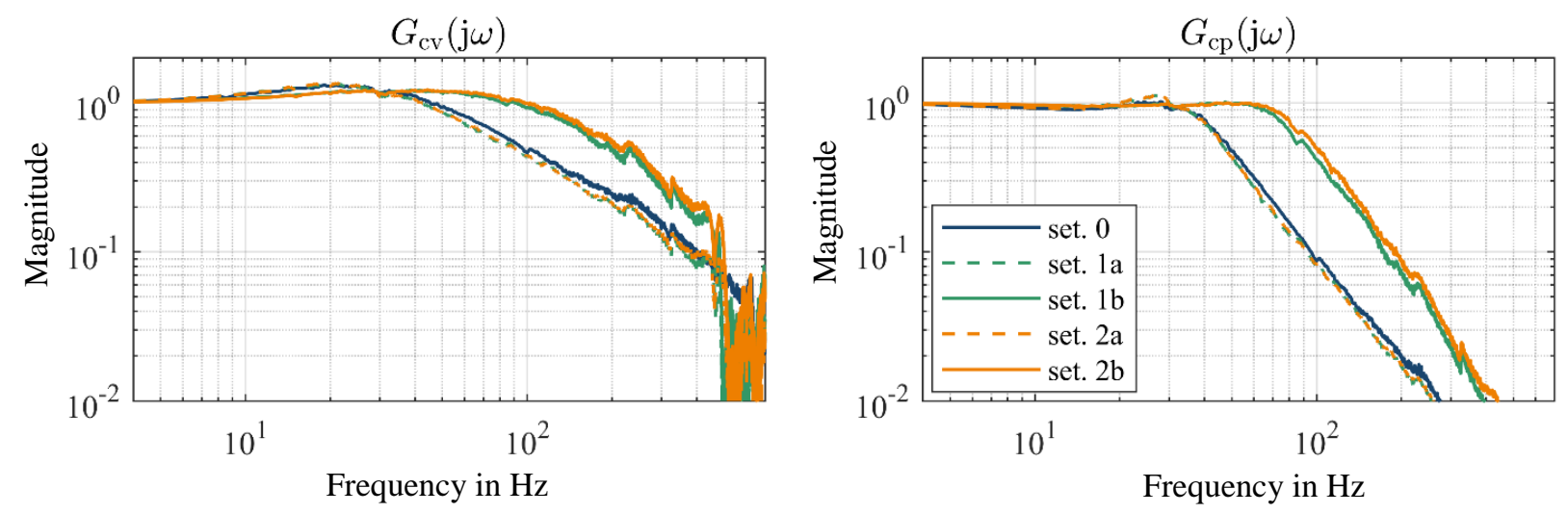

Fig. 11. Frequency response of the closed velocity loop and the closed position loop

\section{SUMMARY}

This study describes the design of compliant elements implemented in a gantry stage equipped with two linear direct drives. The usage of link elements with thin, packaged spring-steel-sheets has the opportunity to adjust the stiffness properties of the joint by varying parameters like the number of steel sheets used in a layered flexible link. The aim is to design compliant hinges with a low rotational stiffness, but with high stiffness-values to other degrees of freedom. With the experimental identification of the FRF Matrix of the multivariable system, the improvement of the stability by the raise of the gain margin of the closed velocity loop is verified. Furthermore, it is possible to increase the gains of the velocity and position control and, therefore, increase the bandwidth of the closed loops. In addition, by releasing the mechanical coupling of the drives, the reaction forces the actuators have to provide can be reduced significantly. Thus, the forces that need to be withstood by the guides is reduced and the durability of the guides can probably be increased.

Further investigations concerning the variation of parameters like length, thickness or the number of sheets enabling optimal joint design for various specific applications, will be subject to future research.

\section{ACKNOWLEDGEMENTS}

This research was supported by a German Research Foundation (DFG) grant, received within the research project "Application potential of articulated coupled drive and guide elements for increase of movement dynamics and accuracy” (IH 124/6-3), which is gratefully acknowledged. 
REFERENCES

[1] BELL D.J., LU T.J., FLECK N.A., SPEARING S.M., 2005, MEMS Actuators and Sensors: Observations on Their Performance and Selection for Purpose, Journal of Micromechanics and Microengineering, 15/7, 153-164.

[2] BHARGAV S.D.B., JORAPUR N., ANANTHASURESH G.K., 2015, Micro-Scale Composite Compliant Mechanisms for Evaluating the Bulk Stiffness of MCF-7 Cells, Mechanism and Machine Theory, 91, 258-268.

[3] SÖNMEZ Ü., 2007, Compliant MEMS Crash Sensor Designs: The Preliminary Simulation Results, IEEE Intelligent Vehicles Symposium.

[4] ELFIZY A.T., BONE G.M., ELBESTAWI M.A., 2005, Design and Control of a Dual-Stage Feed Drive, International Journal of Machine Tools and Manufacture, 45/2, 153-165.

[5] YAO Q., DONG J., FERREIRA P.M., 2007, Design, Analysis, Fabrication and Testing of a Parallel-Kinematic Micropositioning XY Stage, International Journal of Machine Tools and Manufacture, 47/6, 946-961.

[6] KANG D., GWEON D., 2012, Development of Flexure Based 6-Degrees of Freedom Parallel Nano-Positioning System with Large Displacement, Review of Scientific Instruments, 83/3, 035003.

[7] TIAN Y., SHIRINZADEH B., ZHANG D., 2010, Design and Dynamics of a 3-DOF Flexure-Based Parallel Mechanism for Micro/Nano Manipulation, Microelectronic Engineering, 87/2, 230-241.

[8] KIM D., KANG D., SHIM J., SONG I., GWEON D., 2005, Optimal Design of a Flexure Hinge-Based XYZ Atomic Force Microscopy Scanner for Minimizing Abbe Errors, Review of Scientific Instruments, 76/7, 073706.

[9] LI Y., XU Q., 2011, A Totally Decoupled Piezo-Driven XYZ Flexure Parallel Micropositioning Stage for Micro/ Nanomanipulation, IEEE Transactions on Automation Science and Engineering, 8/2, 265-279.

[10] BONO M., HIBBARD R., 2007, A Flexure-Based Tool Holder for Sub- $\mu$ m Positioning of a Single Point Cutting Tool on a Four-Axis Lathe, Precision Engineering, 31/2, 169-176.

[11] CIBLAK N., LIPKIN H., 2003, Design and Analysis of Remote Center of Compliance Structures, Journal of Robotic Systems, 20/8, 415-427.

[12] GIAM T.S., TAN K.K., HUANG S., 2007, Precision Coordinated Control of Multi-Axis Gantry Stages, ISA Transactions, 46/3, 399-409.

[13] SENCER B., MORI T., SHAMOTO E., 2013, Design and Application of a Sliding Mode Controller for Accurate Motion Synchronization of Dual Servo Systems, Control Engineering Practice, 21/11, 1519-1530.

[14] PEUKERT C., PÖHLMANN P., MERX M., MÜllER J., IHLENFELDT S., 2019, Modal-Space Control of a Linear Motor-Driven Gantry System, MM Science Journal, 12/4, 3285-3292.

[15] KOTA S., JOO J., LI Z., RODGERS S.M., SNIEGWSKI J., 2001, Design of Compliant and Mechanisms: Applications and to MEMS, Analog Integrated Circuits and Signal Processing, 29/1-2, 7-15.

[16] PEUKERT C., MERX M., MÜLlER J., IHLENFELDT S., 2017, Flexible Coupling of Drive and Guide Elements for Parallel-Driven Feed Axes to Increase Dynamics and Accuracy of Motion, Journal of Machine Engineering, 17/2, 77-89.

[17] IHLENFELDT S., MÜLLER J., MERX M., PEUKERT C., 2019, Kinematically Coupled Force Compensation Experimental Results and Advanced Design for the 1D-Implementation, Journal of Manufacturing and Materials Processing, 3/24, 1-13.

[18] HOWELL L.L., MAGLEBY S.P., OLSEN B.M., 2013, Handbook of Compliant Mechanisms, John Wiley \& Sons, Chichester.

[19] LOBONTIU N., PAINE J.S.N., GARCIA E., GOLDFARB M., 2001, Corner-Filleted Flexure Hinges, Journal of Mechanical Design, 123/3, 346-352.

[20] HOPKINS J.B., CULPEPPER M.L., 2010, Synthesis of Multi-Degree of Freedom, Parallel Flexure System Concepts Via Freedom and Constraint Topology (FACT) - Part I: Principles, Precision Engineering, 34/2, 259-270.

[21] MACFARLANE A.G.J., POSTLETHWAITE I., 1977, The Generalized Nyquist Stability Criterion and Multivariable Root Loci, International Journal of Control, 25/1, 81-127. 